Комплаентность - определяющий фактор эффективности профилактического применения ацетилсалициловой кислоты

\author{
В.В. Рафальский*, А.Н. Багликов \\ ГОУ ВПО Смоленская государственная медицинская академия Минздравсоцразвития РФ. Смоленск, \\ Россия.
}

\title{
Compliance as the major effectiveness determinant in preventive acetylsalicylic acid therapy
}

\author{
V.V. Rafalskiy*, A.N. Baglikov \\ Smolensk State Medical Academy. Smolensk, Russia
}

\begin{abstract}
Применение ацетилсалициловой кислоты (АСК) у пациентов с сердечно-сосудистыми заболеваниями (СС3), является одним из самых эффективных подходов в профилактике осложнений ССЗ. У значительной части пациентов, отмечается недостаточно высокая комплаентность длительному профилактическому приему АCK (44-71 \%), что сопровождается снижением антитромбоцитарного эффекта препарата и повышением в 2-3 раза риска осложнений, включая инфаркт миокарда (ИМ), ишемический инсульт (ИИ) и др. Улучшить комплаентность пациентов приему АСК возможно за счет улучшения сотрудничества межу врачом и пациентом, информирования и убеждения пациентов в необходимости соблюдения рекомендаций; важное значение имеет назначение кишечнорастворимых форм АСК, улучшающих переносимость препарата. Перспективными являются подходы, основанные на использовании современных упаковок (календарные блистеры) и информационных технологий (телефонные коммуникации, MGMM).
\end{abstract}

Ключевые слова: ацетилсалициловая кислота, комплаентность, сердечно-сосудистые заболевания, профилактика.

\begin{abstract}
Acetylsalicylic acid (ASA) treatment of patients with cardiovascular disease (CVD) is one of the most effective methods for CVD event prevention. In a substantial proportion of the patients, however, long-term preventive ASA treatment compliance is low (44-71\%). Low compliance is associated with reduced antiplatelet effects of ASA and a 2-3-fold increase in the risk of myocardial infarction (MI), ischemic stroke (IS), and other cardiovascular events. ASA therapy compliance could be improved by achieving better collaboration between doctors and patients, while educating patients and explaining the importance of following the doctors' advice. In addition, enteric-coated ASA forms could play an important role in improving treatment tolerability and, therefore, increasing compliance. Other potentially effective measures include the use of modern packaging (calendar blisters) and information technologies (telephone communication, MGMM).
\end{abstract}

Key words: Acetylsalicylic acid, compliance, cardiovascular disease, prevention.

Профилактика и лечение сердечно-сосудистых заболеваний (СС3) являются одними из самых актуальных проблем современного здравоохранения. Рациональная фармакотерапия СС3 включает не только тактику патогенетического лечения больных с их клиническими проявлениями, но и стратегии комплексной и систематической первичной и вторичной профилактики, широкое внедрение которых в развитых странах позволило значительно снизить смертность от сердечно-сосудистых событий (ССС) и увеличить продолжительность жизни [1]. Применение антитромбоцитарных препаратов (АТП) является одним из самых эффективных подходов в профилактике сердечно-сосудистых осложнений (CСO). Долгосрочный и продолжительный прием АТП, согласно международным и отечест-

(с)Коллектив авторов, 2011

E-mail: v.rafalskiy@mail.ru

Тел.: (4812) 681811

Факс: (4812) 315310

[Рафальский В.В. ("контактное лицо) - профессор кафедры управления и экономики фармации, Багликов А.Н. - ординатор кафедры клинической фармакологии]. 
венным рекомендациям (ВНОК 2009, ESC 2007), показан всем больным ишемической болезнью сердца (ИБС) с целью улучшения прогноза и снижения сердечно-сосудистого риска (ССР) [2,3]. Ацетилсалициловую кислоту (АСК) как АТП начали использовать еще в 70-х годах прошлого столетия [4]. Однако за 40 лет применения препарат не только не утратил своего клинического значения, но до настоящего времени остается наиболее широко распространенным препаратом для профилактики тромбозов.

Опубликованные в 1994 и 2002 гг. мета-анализы, подготовленные международной группой ученых, известных как Antitrombotic Trialists Collaboration, оценили результаты 145 и 287 рандомизированных, клинических исследований (РКИ), соответственно, с участием > 200 тыс. пациентов. Оба мета-анализа убедительно показали, что АCK достоверно снижает риск рецидивов ССО у лиц, перенесших в прошлом инфаркт миокарда (ИМ) и ишемический инсульт (ИИ), а также страдающих другой сердечно-сосудистой патологией: стенокардия, мерцательная аритмия, хирургические сосудистые заболевания и др. [5,6].

Таким образом, основные аспекты профилактического эффекта АСК были достаточно подробно изучены уже в 90-х годах прошлого века. Вместе с тем, в тот же период остро обозначилась проблема неадекватного использования АСК пациентами, которым был назначен препарат для длительного профилактического приема, другими словами, проблема низкой комплаентности пациентов долгосрочной терапии АСК.

В настоящее время проблема комплаентности при длительном приеме лекарственных препаратов (ЛП) занимает одно из центральных мест в рациональной фармакотерапии хронических заболеваний. Термин “комплаентность" (“compliance", “приверженность”) был предложен еще в 70-х годах прошлого столетия [7]. В контексте оказания медицинской помощи он означает степень соответствия поведения больного (в отношении приема ЛП, соблюдения диеты, изменения образа жизни и др.) рекомендациям, полученным от врача [8]. То, что комплаентность к той или иной терапии является, безусловно, ключевым звеном между процессом лечения и его исходом, отчетливо понимают как врачи, так и пациенты. Несмотря на это, данные Всемирной организации здравоохранения (ВО3) свидетельствуют о том, что длительное соблюдение назначений врача при хронических заболеваниях наблюдается лишь в $50 \%$ случаев [9].

Впервые о проблеме недостаточной приверженности пациентов длительному приему АCK, назначаемому для вторичной профилактики ССО широко заговорили после проведенного в 1995 г. популяционного исследования Nova Scotia Health Service Survey. Результаты этого исследования пока- зали, что более половины всех обследованных больных, страдающих различными СС3, не соблюдали предписанный режим приема АСК. Установлено, что на момент обследования больные с перенесенным ИМ принимали АСК в 55 \% случаев, с ИИ в $47 \%$, с установленным диагнозом ИБС - в $54 \%$. Важно отметить, что исключение из статистического анализа лиц, которым прием АСК был противопоказан, достоверно не изменило эти показатели. Таким образом, в обследованной популяции только $53 \%$ пациентов с СС3 принимали АСК с целью профилактики [10].

Несмотря на доказанную эффективность и необходимость приема АСК лицами с ССР, низкая комплаентность терапии вплоть до полного отказа, нередко наблюдаются в клинической практике. Оценка североамериканскими учеными в течение года приверженности пациентов с коронарными заболеваниями длительному приему сердечно-сосудистых ЛП, назначенных при выписке из стационара, показала, что из 1326 пациентов, включенных в исследование, только $54 \%$ соблюдали назначения врача и регулярно в течение года принимали все предписанные ЛП. При этом отмена приема АСК по разным причинам после года терапии имела место в $18 \%$ случаях [11].

В когортном исследовании была оценена приверженность пациентов, перенесших ИМ, длительной комбинированной медикаментозной терапии после выписки из стационара [12]. Анализ полученных данных показал, что к 120 дню наблюдения только 74 \% пациентов соблюдали предписанный режим лечения. Причем, чаще всего, пациенты отказывались от приема “некардиологических" ЛП (66 \%), а “кардиологические” ЛП принимали 82,3 \% пациентов. Приверженность длительному приему ACK была самой низкой - 44,02 \% пациентов к 120 дню наблюдения по разным причинам приостановили или полностью отменили прием этого ЛП.

При ретроспективной оценке приверженности длительному приему ЛП, в т.ч. АСК, у 30 тыс. пациентов, страдающих СС3 в течение $\geq 7$ лет, установлено, что ежедневный профилактический прием ACK на протяжении всего периода наблюдения соблюдали только 71 \% пациентов [13].

В 2006г были опубликованы результаты крупнейшего международного, обсервационного исследования REACH (REduction of Atherothrombosis for Continued Health) Registry, включившего 67888 пациентов из 44 стран [14]. Главной целью проекта REACH Registry была глобальная оценка распространенности, факторов риска (ФР), смертности пациентов с атеротромботическими заболеваниями - ИМ, ИИ, нестабильная стенокардия (НС), тромбозы периферических артерий и др., а также разработка мероприятий, направленных на снижение заболеваемости и повышение выживаемости пациентов с атеротромбозом. В исследовании был про- 
Влияние приверженности длительному профилактическому приему АСК на клинические исходы

\begin{tabular}{|c|c|c|c|}
\hline Автор, год & Дизайн исследования & $\mathrm{n}$ & Основные результаты \\
\hline Krumholz H, et al, 1996 [15] & Обсервационное исследование & 4149 & $\begin{array}{l}\text { Риск сердечно-сосудистой смерти у пациентов, приверженных } \\
\text { приему АСК достоверно ниже, чем у пациентов, регулярно } \\
\text { не принимавших АСК (OP - 0,77; } 95 \text { \% ДИ }[0,61-0,98]) .\end{array}$ \\
\hline Ferrari E, et al, 2004 [16] & $\begin{array}{l}\text { Проспективное, обсервационное, } \\
\text { исследование (анкетирование) }\end{array}$ & 1236 & $\begin{array}{l}\text { У пациентов, не соблюдавших прием АСК, в 13,3 \% случаях } \\
\text { развивались ССО. }\end{array}$ \\
\hline Cotter G, et al, 2004 [19] & $\begin{array}{l}\text { Проспективное, обсервационное, } \\
\text { когортное исследование }\end{array}$ & 95 & $\begin{array}{l}\text { Частота неблагоприятных исходов и госпитализаций в гр. при- } \\
\text { верженных лечению АСК пациентов - } 6 \text { \% и } 11 \text { \% соответствен- } \\
\text { но, в гр. “неприверженных" - } 42 \text { \% и } 67 \% \text {. }\end{array}$ \\
\hline Iakovou I, et al, 2005 [17] & $\begin{array}{l}\text { Проспективное, обсервационное, } \\
\text { когортное исследование }\end{array}$ & 2229 & $\begin{array}{l}\text { Риск тромбоза стента и ИМ у лиц, перенесших ЧКВ и прини- } \\
\text { мающих АСК, ниже, чем у пациентов, не соблюдающих прием } \\
\text { ACК - ОР 89,78; } 95 \text { \% ДИ [29,90-269,60] (p<0,001) }\end{array}$ \\
\hline Newby L, et al, 2006 [13] & $\begin{array}{l}\text { Проспективное, обсервационное, } \\
\text { когортное исследование }\end{array}$ & 31750 & $\begin{array}{l}\text { Риск сердечно-сосудистой смерти у пациентов, приверженных } \\
\text { приему АСК значительно ниже, чем у неприверженных пациен- } \\
\text { тов - OР - 0,58; } 95 \text { \% ДИ [0,54-0,62] }\end{array}$ \\
\hline $\begin{array}{l}\text { Biondi-Zoccai G, et al, } 2006 \\
\text { [21] }\end{array}$ & Мета-анализ 6 исследований & 50279 & $\begin{array}{l}\text { У пациентов с низкой приверженностью лечению АСК риск } \\
\text { развития ССО выше по сравнению с приверженными пациента- } \\
\text { ми }-\mathrm{OP}=3,14 \text { ДИ } 95 \%[1,75-5,61](\mathrm{p}=0,0001)\end{array}$ \\
\hline
\end{tabular}

веден анализ фармакотерапии пациентов с атеротромботическими заболеваниями, который показал, что большинство врачей разных стран при выборе лекарственной терапии руководствуются современными научными рекомендациями, назначая пациентам с профилактической целью длительный прием АСК. Однако, несмотря на то, что прием АСК был рекомендован 78,6 \% пациентов, хорошая приверженность была отмечена только у 67,4 \% больных.

Таким образом, в настоящее время накопилось достаточно данных, позволяющих утверждать, что у значительной части пациентов, которым показан длительный профилактический прием АСК, отмечается недостаточно высокая комплаентность этому ЛП. Чаще всего низкая комплаентность может выражаться либо в полном отказе пациента от приема, либо в нарушении режима приема ЛП. Однако, как бы ни были очевидны, медицинские последствия низкой комплаентности, тем не менее, для ее строгой объективной оценки, необходимо обратиться к результатам клинических исследований.

В настоящее время опубликованы как результаты отдельных исследований, так и мета-анализы, посвященные изучению отдаленных исходов у пациентов с низким уровнем комплаентности приему АCK (таблица 1). Показано, что низкая приверженность профилактическому приему АСК у больных, перенесших острый коронарный синдром (ОКС), ассоциируется с повышением риска повторного возникновения ССО, включая ИМ, ИИ, госпитализации по поводу НС.

При наблюдении за 4149 пациентами, перенесшими ИМ, было установлено, что назначение и прием АСК после выписки из стационара значительно влияет на смертность [15]. Через 6 мес наблюдения риск смерти у больных, регулярно принимавших АCK, оказался достоверно ниже даже после поправки на демографические, клинические факторы и учета сопутствующей терапии - отношение рисков (ОР) - 0,77; ДИ 0,61-0,98 [15]. В исследовании 2006г было установлено, что риск сердечно-сосудистой смерти у пациентов, приверженных приему АСК, значительно ниже, чем у пациентов с низкой комплаентностью (ОР - 0,58; $95 \%$ ДИ 0,54-0,62) [13].

При опросе 1236 пациентов, госпитализированных в стационар с установленным диагнозом ОКС, выявлено, что 13,3 \% пациентов с рецидивом коронарного события в течение $\geq 1$ мес. не принимали АСК. Примечательно, что период от момента отмены приема АСК до развития рецидива ОКС составил в среднем $10 \pm 1,9$ дней [16].

Развитие ССО, в т.ч. фатального и нефатального ИМ у пациентов, перенесших чрескожное коронарное вмешательство (ЧКВ), было изучено в проспективном когортном исследовании [17]. В исследование были включены 2229 пациентов, перенесших ЧКВ (стентирование, шунтирование и др.) и длительно принимавших АСК. Уже после 9 мес. наблюдения у 58 пациентов были выявлены различные ССО, связанные с повышенным тромбообразованием - ИМ, тромбоз стента и др. При этом высокий риск развития ССО в значительной степени коррелировал с низкой комплаентностью приему ACK: у подавляющего большинства пациентов с ССО была зафиксирована приостановка или полная отмена приема АСК: ОР - 89,8; 95 \% ДИ 29,9-269,6 ( $<<0,001)$. Аналогичное исследование показало, что раннее, в течение первых 48 ч, назначение ACK для длительного приема пациентам, перенесшим аортокоронарное шунтирование (АКШ), позволяет на $48 \%$ снизить риск развития ИМ, на 50 \% - МИ. Риск фатальных ССО у пациентов, не соблюдавших по различным причинам предписанный режим приема АСК, был почти в 2 раза выше, чем у пациентов, принимавших ACK, $-4,0 \%$ и $1,3 \%$, соответственно $(\mathrm{p}<0,001)$ [18]. 
Особый интерес представляет исследование израильских ученых, направленное на выяснение связи между комплаентностью длительному приему АСК и “резистенстностью” к АСК. В этом исследовании пациенты, перенесшие ИМ, были разделены на три группы (гр.): “приверженные”, “неприверженные” (не выполняющие рекомендации врача) и “резистентные к АCK” (больные, у которых, несмотря на регулярный прием АСК, отсутствовало влияние препарата на тромбоциты) [19]. Установлено, что после 12 мес. наблюдения частота ССО - смерть, повторный ИМ, госпитализация по поводу НС, в разных гр. отличалась. Частота ССО и госпитализаций у “неприверженных” больных оказалась весьма значительной - 42 \% и $67 \%$, соответственно, в то же время, эти показатели у “резистентных” были значительно меньше - $11 \%$ и $11 \%$, а в гр. “приверженных" пациентов - $6 \%$ и $11 \%$, соответственно. Эти результаты свидетельствуют не только о большом значении регулярного приема АСК для реализации его профилактического эффекта, но и об относительности понятия “резистентность к АСК” и многообразии механизмов превентивного эффекта ЛП.

В 2010г опубликован дополнительный анализ хорошо известного исследования Physicians' Health Study, в котором оценивали возможные причины неэффективности профилактического приема АСК. Было продемонстрировано, что основной причиной является низкая комплаентность - пациенты, принявшие за год > 150 таблеток АСК, имели статистически значимое снижение риска ИМ в сравнении с плацебо - ОР - 0,55; 95 \% ДИ 0,44-0,70 (p $\leq 0,0001)$, а у пациентов, принявших за год $<150$ таблеток ACK, риск ИМ не отличался от плацебо [20].

Особый интерес представляют результаты метаанализов, объединяющих результаты нескольких исследований. В 2006г итальянскими учеными был опубликован мета-анализ, объединивший результаты 6 многоцентровых, проспективных, когортных исследований и данные наблюдения за 50279 пациентами, перенесшими эпизод ОКС или кардиохирургическое вмешательство, и длительно принимавшими АСК для профилактики ССО. Результаты исследования убедительно показали, что пациенты с низкой приверженностью длительному приему ACK имеют значительно более высокий риск развития ССО по сравнению с пациентами, следующими всем предписанным рекомендациям - OP - 3,1; ДИ $95 \%$ [1,75-5,61] (p=0,0001) [21].

Таким образом, вопрос высокой приверженности длительному профилактическому приему $\mathrm{ACK}$ - это в итоге вопрос достижения и сохранения эффективности АТП. Соответственно, выявление и изучение факторов, влияющих на комплаентность - дополнительная возможность повысить эффективность профилактического приема АСК. Вопрос о том, какие факторы лежат в основе низкой при- верженности терапии АСК, широко обсуждается в литературе > 40 лет. Однако, несмотря на значительный объем проведенных к настоящему времени исследований, значительных достижений в данной области сделано не было. Одной из основных причин является отсутствие объективного метода измерения комплаентности, а, следовательно, единства в понимании этой проблемы.

Данные зарубежных исследований, посвященных изучению комплаентности пациентов с CC3 рекомендованной терапии, свидетельствуют о существовании как минимум 3 гр. факторов, оказывающих влияние на приверженность пациентов предписанному лечению [22]. I гр. - социальноэкономические факторы. Они обуславливают несоблюдение рекомендаций в силу неспособности пациентов обеспечить себя необходимыми ЛП. К этой гр. факторов относится безработица, ранний выход на пенсию, инвалидность, определяющие низкий уровень жизни, плохо развитую систему медицинского страхования и многое другое (рисунок 1). Исследования показали, что социальноэкономические факторы в сочетании с низким уровнем грамотности пациентов с СС3 способствуют значительному снижению приверженности длительной медикаментозной терапии [23].

II гр. факторов относится к эффективности коммуникативных связей в сфере врач - пациент. Наличие эффективной коммуникации является неотъемлемым условием соблюдения и выполнения пациентами предписанных врачом рекомендаций. Основные трудности, способствующие снижению эффективности сотрудничества между врачом и пациентом, а, следовательно, несоблюдению больными назначенного режима медикаментозной терапии, связаны с так называемыми коммуникативными барьерами: низкий уровень образования, безграмотность, языковые и культурные барьеры, злоупотребление алкоголем и психоактивными веществами, депрессивные состояния, психические заболевания и др. [13].

III гр. факторов связана с мотивацией пациентов. Плохая информированность пациентов относительно их заболевания, недооценка тяжести болезни, а также непонимание пользы от получаемого лечения приводят к снижению мотивации, и в конечном итоге к нарушению регулярного приема ЛП, вплоть до полного отказа. Эта проблема часто усугубляется страхом побочных явлений (ПЯ) и токсичности ЛП. Пациенты с меньшей вероятностью используют препараты, длительный прием которых ассоциируется с частыми ПЯ, а также не коррелирует с выраженным и быстро развивающимся эффектом. В отличие от таких ЛП, как анальгетики, антибактериальные средства и др., АСК, как и многие другие кардиологические ЛП, применяется с превентивной целью, что не позволяет пациентам объективно оценить эффект вследствие отсутствия 


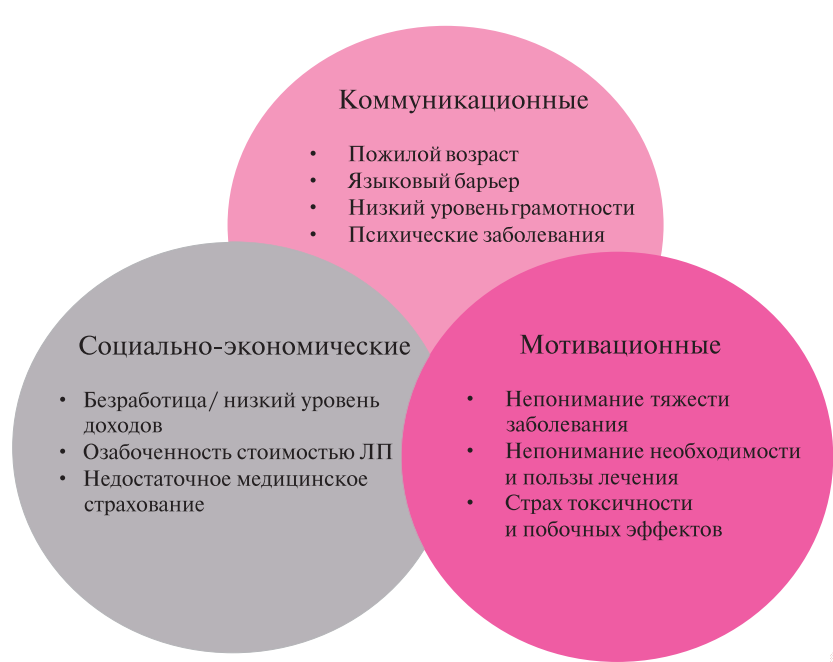

Puc. 1 Причины низкой приверженности пациентов длительной фармакотерапии СС3 [22].

быстрого изменения качества жизни (КЖ) [22].

Анализ возможных причин низкой приверженности приему “кардиологических” ЛП, в т.ч. АСК, показал, что такие факторы, как низкий уровень доходов, возраст, взаимоотношения пациента с врачом, объем сопутствующей терапии (количество принимаемых препаратов, в т.ч. “некардиологических") оказывают существенное влияние на комплаентность. Установлено, что в целях повышения приверженности терапии для таких пациентов целесообразно проведение консультативных мероприятий, информирующих пациентов об успешности долгосрочной терапии препаратами АСК [12].

Учитывая сказанное выше, становится очевидно, что в связи с высокой распространенностью СС3 и необходимостью широкого применения АCK как профилактического средства, выработка рекомендаций, направленных на повышение компалентности пациентов к длительному приему АCK, является первоочередной задачей клинической фармакологии. В зарубежной литературе появляется все больше сведений, демонстрирующих важность информирования пациентов о деталях заболевания, его возможных осложнениях, об основных принципах и видах лечения в целях повышения приверженности терапии. Обсуждается необходимость рационализации подходов к назначению ЛП, включающих не только научно-доказательную обоснованность применения ЛП, но и убеждение пациентов в успешности проводимого лечения посредством информирования, регулярного напоминания о приеме ЛП, контроля за процессом лечения и соблюдением пациентами рекомендаций [24]. Данные меры, по мнению авторов, способствуют улучшению сотрудничества между врачом и пациентом, что в конечном итоге может способствовать повышению комплаентности долгосрочному лечению и, вследствие этого, улучшению исходов терапии.
В контексте обсуждения мер по улучшению комплаентности пациентов длительной фармакотерапии, целесообразно обратиться к результатам соответствующих исследований. В 2007-2008 гг. было выполнено исследование АСТ (Aspirin underutilization and compliance in Cardiovascular diseases Treatment), направленное на изучение практики назначения врачами АСК для профилактики ССО, а также выработку мер по улучшению комплаентности пациентов лечению. В исследовании приняли участие 7363 врачей из 18 стран Европы, Латинской Америки и Азии, в т.ч. Российской Федерации $[25,26]$. Результаты опроса показали, что большинство врачей согласны с современными научными представлениями о профилактической роли АCK у больных СС3. Длительная терапия АСК была рекомендована в среднем $>90 \%$ пациентов. АСК не назначали в связи с непереносимостью и ожиданием нежелательных лекарственных реакций (НЛР). Следует отметить, что в России длительный прием АСК был рекомендован в среднем 84 \% больных, что несколько меньше, чем в других странах (к примеру, в Швейцарии - 93 \%, в Германии - 92 \%) [26]. Основной причиной отказа от назначения препарата было опасение НЛР (24\%).

Комплаентность пациентов лечению в целом была расценена как недостаточная [25,26]. В Европе полностью привержены профилактическому приему АСК были $63 \%$ больных. В странах Латинской Америки и Азии хорошая приверженность лечению была отмечена лишь у $54 \%$ и 55 \% больных, соответственно. В России показатели приверженности были сопоставимы с европейскими (59\%) $[25,26]$.

В ходе исследования врачам предлагалось оценить по 10-балльной шкале эффективность различных способов улучшения отношения пациентов с СС3 к долгосрочному лечению препаратами АСК. По этому вопросу мнения врачей разных стран в значительной степени совпали. Наиболее эффективными (> 7 баллов) признаны использование материалов, демонстрирующих профилактическую эффективность длительного приема АСК и, в особенности, совместная договоренность врача и пациента по этому вопросу. Примечательно, что российские врачи сочли высокоэффективными (>7баллов) достаточно большое число мероприятий, направленных на повышение приверженности пациентов приему АСК. Среди прочих российские коллеги отдавали предпочтение мероприятиям по организации круглых столов совместно с другими врачами по обмену опытом о долгосрочной терапии препаратами ACK (72 \%), использованию квалифицированной информации для врачей по убеждению “трудных пациентов”, которые не придерживаются долгосрочной терапии (73\%), а также отчетов научных исследований, доказывающих эффективность долгосрочной терапии АСК (74 \%) [26]. 
Таким образом, очевидно, что успех длительного профилактического лечения АСК во многом определяется сотрудничеством между врачом и пациентом для формирования совместного решения по поводу долгосрочной терапии. Это означает, что требуемый уровень научных знаний современного врача должен быть достаточным не только для формирования собственного убеждения, но и для возможности предоставления необходимой информации пациентам. Только в этом случае врач способен грамотно назначить терапию и убедить пациентов в необходимости соблюдения рекомендаций.

Серьезной проблемой, препятствующей обеспечению соответствующей комплаентности длительному лечению, является опасения врачей и пациентов в связи с возможным развитием НЛР на АСК. По данным исследования АСТ ожидание НЛР АСК стало одной из основных причин отказа от назначения $\mathrm{ACK} \geq 25 \%[25,26]$. Наиболее частой НЛР, связанной с приемом АСК, является гастротоксичность - нестероидные противовоспалительные средства (НПВС)-гастропатия. Жалобы со стороны желудочно-кишечного тракта (ЖКТ) - изжога, тошнота, рвота, боли в эпигастрии и др., при длительном приеме АСК могут наблюдаться у $25 \%$ больных [27]. Частота развития более опасной формы НПВС-гастропатии - желудочно-кишечного кровотечения (ЖКК), при длительном приеме АСК составляет 2-3\% [28].

В недавно опубликованном, многоцентровом, обсервационном исследовании авторы изучали влияние частоты развития гастротоксических эффектов АCK на комплаентность длительному лечению у пациентов с СС3 [29]. В исследовании приняли участие 340 пациентов. После 3 мес. наблюдения оказалось, что 18 \% пациентов плохо соблюдали предписанный режим лечения, а в $13 \%$ случаев прием АСК был полностью отменен. Анализ полученных данных показал, что низкая приверженность лечению АСК коррелировала с частотой развития НПВС-гастропатии. Риск отмены АСК был значительно выше у пациентов, отмечавших в течение нед возникновение, как минимум, 3 эпизодов желудочно-кишечной (ЖК) симптоматики в сравнении с пациентами, не предъявлявшими жалоб со стороны ЖКТ - ОР = 7,52; 95 \% ДИ 2,57-22,04).

В связи с этим одним из возможных путей, направленных на повышение безопасности и переносимости, а, следовательно, комплаентности лечению ACK, рассматривается разработка желудочно-резистентных лекарственных форм АСК. Среди них наиболее рациональным представляется выбор, так называемых, кишечнорастворимых форм АCK (Аспирин®Кардио, Байер Шеринг Фарма, Германия), особенности технологии которых способствуют растворению и высвобождению АСК в щелочной среде 12-перстной кишки, снижая таким образом риск повреждающего действия АСК на слизистую оболоч- ку желудка. Многочисленные исследования безопасности и переносимости кишечнорастворимых форм ACK с использованием эндоскопии убедительно показали достоверное снижение риска развития эрозивно-язвенных поражений ЖКТ, включая "большие” (ЖКК) и “малые” (изжога, диспепсия) НЛР, в результате приема АСК [30]. К сожалению, подобных исследований крайне мало, цель большинства опубликованных в настоящее время исследований сравнение частоты развития “больших" проявлений НПВС-гастропатии (кровотечение, язва) у пациентов, принимающих различные лекарственные формы АСК. В связи с этим особенный интерес представляет исследование отечественных ученых, посвященное оценке переносимости, безопасности и влияния на показатели КЖ разных лекарственных форм АСК, поскольку эти факторы непосредственно влияют на комплаентность АСК [31]. В проспективном исследовании участвовали 120 пациентов, перенесших ИМ, которые были разделены на 4 сопоставимые по возрасту и полу гр. для приема Аспирина®Кардио 100 мг(Байер Шеринг Фарма, Германия), ТромбоАсса 100 мг - кишечнорастворимые лекарственные формы АСК, Кардиомагнила 75 мг (буферизованная лекарственная форма АCK) и АCK 125 мг. Через 12 мес. наблюдения частота развития диспепсических симптомов в гр. Аспирина®Кардио составила 6,6 \%, в гр ТромбоАсса - 19,9\%, в гр. Кардиомагнила - 16,6 \%, в гр АCK - 23,3\%.

По результатам эндоскопического исследования (ЭФГДС) наибольшая частота эрозивного поражения слизистой оболочки желудка через 12 месяцев лечения выявлялась в гр ТромбоАсса и АСК - 13,3\% и 23,3\%, соответственно; в гр. Кардиомагнила эндоскопическая картина НПВП-гастропатии наблюдалась у 9,9 \% пациентов. Использование Аспирина ${ }^{\circledR}$ Кардио 100 мг/сут. в течение 12 мес. по данным ЭФГДС сопровождалось наименьшей частотой развития НПВП-гастропатии - 3,3 \% [31]. Таким образом, кишечнорастворимые формы АСК способствуют улучшению переносимости, изменению КЖ пациентов, которые являются критическими для соблюдения комплаентности длительному приему препарата и, в итоге, для достижения целей антитромбоцитарной терапии.

По данным зарубежной литературы на комплаентность пациентов существенное влияние может оказывать не только использование лекарственных форм с улучшенными фармакокинетическими свойствами, но также особенности упаковки ЛП, различные модификации которой обеспечивают простоту и удобство приема. Влияние упаковки на комплаентность пациентов было изучено в нескольких проспективных, контролируемых исследованиях.

В 1991г была опубликована работа, в которой сравнивали результаты наблюдения за 84 пожилыми пациентами, разделенными на две сопоставимые гр. 
Пациенты первой гр. принимали препарат из обычного флакона, а пациенты второй гр. - из календарного блистера [32]. Уже на 10 день после выписки из стационара было установлено, что комплаентность пациентов в гр. с использованием календарного блистера значительно выше, чем в гр. с приемом препарата из обычного флакона - 68,8 \% и 41,0 \%, соответственно, через 3 мес. эти показатели составили 48,9\% и 23,1 \%, соответственно. Также было показано, что только переход на использование календарных блистеров позволяет в короткие сроки значительно снизить индекс “некомплаентности” пациентов с 9,17 до 2,04 по сравнению с приемом препарата из флаконов [33]. Таким образом, еще одним подходом, позволяющим повысить комплаентность у пациентов, нуждающимся в длительном приеме ACK, является рациональный выбор упаковки ЛП. В связи с этим очень потенциальным представляется применение новой упаковки препарата Аспирина ${ }^{\circledR}$ Кардио с календарным блистером, появившемся с 2010 г. в России. Удобство применения и возможность постоянного самоконтроля помогает пациентам быть более комплаентными и как следствие обеспечивать себе более эффективное лечение.

Еще одним из перспективных направлений повышения комплаентности служит использование современных информационно-компьютерных технологий. Относительно недавно описан так называемый “фармакологический менеджмент” (mesurement-guided medication management, MGMM). Этот подход можно определить как вмешательство, модифицирующее приверженное поведение посредством обеспечения обратной связью с приемом ЛП. Также разработана и применяется в клинических исследованиях технология, которая базируется на использовании электронных датчиков, которые позволяют регистрировать точную дату и время вскрытия флакона или блистера с последующей автогенерацией отчета о приеме препарата и предложением индивидульных рекомендаций для запоминания о приеме ЛП в определенное время сут [34]. Однако сегодня отсутствуют доказательства улучшения клинических исходов вследствие применения программ MGMM, что связано с направленностью исследований в первую очередь на определение факторов, улучшающих комплаентность.

Перспективным может оказаться метод, связанный с использованием телефонных коммуникаций, благодаря услугам которых представляется возможным осуществление мониторинга за процессом лечения и соблюдением пациентами рекомендаций. Применение услуги телефонных коротких текстовых сообщений - SMS (short message service), информирующих пациентов о приеме ЛП, дозе, кратности и других деталях, позволяет предупредить пропуск приема назначенного ЛП и, следовательно, улучшить комплаентность пациентов лечению [35]. Установлено, что приверженность терапии в гр. с применением технологии текстовых SMS сообщений была значительно выше, чем в контрольной гр. $-61,5 \%$ и 49, $8 \%$, соответственно. Систематический обзор 25 КИ (5 нерандомизированных и 20 РКИ) с участием 38060 пациентов [36] убедительно показал, что применение подходов мониторинга, менеджмента и образования, осуществляемых посредством телефонных коммуникаций (голосовых voice mail) или текстовых - SMS) в сочетании со стандартными методами наблюдения и лечения позволяют значительно повысить комплаентность пациентов и, следовательно, качество и эффективность проводимой терапии.

\section{Заключение}

Применение АСК у пациентов с СС3, безусловно, является одним из самых эффективных подходов в профилактике ССО. В настоящее время у значительной части пациентов, отмечается недостаточно высокая комплаентность длительному профилактическому приему АСК (44-71 \%), что наиболее часто выражается либо в полном отказе пациента от приема ЛП, либо в нарушении режима приема. В клинических исследованиях установлено, что низкая приверженность длительному приему АCK сопровождается снижением антитромбоцитарного эффекта препарата и повышением в 2-3 раза риска ССО, включая ИМ, ИИ и др.

Улучшить приверженность пациентов долгосрочному лечению АCK возможно за счет улучшения сотрудничества межу врачом и пациентом, информирования и убеждения пациентов в необходимости соблюдения рекомендаций. Важное значение для повышения комплаентности может иметь использование кишечнорастворимых форм АCK, которые улучшают переносимость препарата. Перспективными являются подходы, основанные на использовании современных упаковок (календарные блистеры) и информационных технологий (телефонные коммуникации, MGMM).

\section{Литература}

1. Capewell S, O'Flaherty M, Ford ES, et al. Potential reductions in United States coronary heart disease mortality by treating more patients. Am J Cardiol 2009; 103: 1703-9.

2. Рекомендации по рациональной фармакотерапии больных сердечно-сосудистыми заболеваниями. Всероссийское научное общество кардиологов. Москва 2009.

3. Rapezzi C, Biagini E, Branzi A. Guidelines for the diagnosis and treatment of non-ST-segment elevation acute coronary syndromes: the task force for the diagnosis and treatment of non-ST-segment elevation acute coronary syndromes of the European Society of Cardiology. Eur Heart J 2008; 29: 277-8.

4. Weiss HJ, Aledort LM. Impaired platelet-connective-tissue reaction in man after aspirin ingestion. Lancet 1967; 2: 495-7. 
5. Collaborative overview of randomised trials of antiplatelet therapy--II: Maintenance of vascular graft or arterial patency by antiplatelet therapy. Antiplatelet Trialists' Collaboration. BMJ 1994; 308: 159-68.

6. Collaborative meta-analysis of randomised trials of antiplatelet therapy for prevention of death, myocardial infarction, and stroke in high risk patients. BMJ 2002; 324: 71-86.

7. Marston MV. Compliance with medical regimens: a review of the literature. Nurs Res 1970; 19: 312-23.

8. Urquhart J. Pharmacoeconomic consequences of variable patient compliance with prescribed drug regimens. Pharmacoeconomics 1999; 15: 217-28.

9. World Health Organization. Adherence to long-term therapies: evidence for action. Geneva: WHO 2003.

10. Rojas-Fernandez CH, Kephart GC, Sketris IS, et al. Underuse of acetylsalicylic acid in individuals with myocardial infarction, ischemic heart disease or stroke: data from the 1995 populationbased Nova Scotia Health Survey. Can J Cardiol 1999; 15: 291-

11. Kulkarni SP, Alexander KP, Lytle B, et al. Long-term adherence with cardiovascular drug regimens. Am Heart J 2006; 151: 18591.

12. Jackevicius CA, Li P, Tu JV. Prevalence, predictors, and outcomes of primary nonadherence after acute myocardial infarction. Circulation 2008; 117: 1028-36.

13. Newby LK, LaPointe NM, Chen AY, et al. Long-term adherence to evidence-based secondary prevention therapies in coronary artery disease. Circulation 2006; 113: 203-12.

14. Bhatt DL, Steg PG, Ohman EM, et al. International prevalence, recognition, and treatment of cardiovascular risk factors in outpatients with atherothrombosis. JAMA 2006; 295: 180-9.

15. Krumholz HM, Radford MJ, Ellerbeck EF, et al. Aspirin for secondary prevention after acute myocardial infarction in the elderly: prescribed use and outcomes. Ann Intern Med 1996; 124: 292-8.

16. Ferrari E, Benhamou M, Cerboni P, et al. Coronary syndromes following aspirin withdrawal: a special risk for late stent thrombosis. JACC 2005; 45: 456-9.

17. Iakovou I, Schmidt T, Bonizzoni E, et al. Incidence, predictors, and outcome of thrombosis after successful implantation of drugeluting stents. JAMA 2005; 293: 2126-30.

18. Mangano DT. Aspirin and mortality from coronary bypass surgery. N Engl J Med 2002; 347: 1309-17.

19. Cotter G, Shemesh E, Zehavi M, et al. Lack of aspirin effect: aspirin resistance or resistance to taking aspirin. Am Heart $\mathbf{J}$ 2004; 147: 293-300.

20. Hennekens CH, Schneider WR, Hebert PR, et al. Hypothesis formulation from subgroup analyses: nonadherence or nonsteroidal anti-inflammatory drug use explains the lack of clinical benefit of aspirin on first myocardial infarction attributed to „aspirin resistance“. Am Heart J 2010; 159: 744-8.
21. Biondi-Zoccai GG, Lotrionte M, Agostoni P, et al. A systematic review and meta-analysis on the hazards of discontinuing or not adhering to aspirin among 50,279 patients at risk for coronary artery disease. Eur Heart J 2006; 27: 2667-74.

22. Baroletti S, Dell'Orfano H. Medication adherence in cardiovascular disease. Circulation 2010; 121: 1455-8.

23. Oates DJ, Paasche-Orlow MK. Health literacy: communication strategies to improve patient comprehension of cardiovascular health. Circulation 2009; 119: 1049-51.

24. Haynes RB, Yao X, Degani A, et al. Interventions to enhance medication adherence. Cochrane Database Syst Rev 2005; 4: CD000011.

25. Zaninelli A, Hu DY, Kaufholz C, et al. Physicians' attitudes toward post-MI aspirin prophylaxis: findings from an online questionnaire in Asia-Pacific. Postgrad Med 2010; 122: 108-17.

26. Zaninelli A, Kaufholz C, Schwappach D. Physicians` attitudes toward post-MI aspirin prophylaxis: findings from an online questionnaire in Europe and Latin America. Postgrad Med 2009; 121: 44-53.

27. Lanas A. Cyclo-oxygenase-1/cyclo-oxygenase-2 non selective non-steroidal anti-inflammatory drugs: epidemiology of gastrointestinal events. Dig Liver Dis 2001; 33 Suppl 2: S29-34.

28. Roderick PJ, Wilkes HC, Meade TW. The gastrointestinal toxicity of aspirin: an overview of randomised controlled trials. $\mathrm{Br} \mathbf{J}$ Clin Pharmacol 1993; 35: 219-26.

29. Pratt S, Thompson VJ, Elkin EP, et al. The impact of upper gastrointestinal symptoms on nonadherence to, and discontinuation of, low-dose acetylsalicylic acid in patients with cardiovascular risk. Am J Cardiovasc Drugs 2010; 10: 281-8.

30. Dietz R, Rauch B. Leitlinie zur Diagnose und Behandlung der chronischen koronaren Herzerkrankung der Deutschen Gesellschaft fur Kardiologie--Herz und Kreislaufforschung (DGK). Z Kardiol 2003; 92: 501-21.

31. Булахова Е.Ю., Кореннова О.Ю., Козырева В.А. и др. Антиагрегантная терапия больных с ишемической болезнью сердца. Справ поликлин врача 2009; 9: 26-30.

32. Ware GJ, Holford NH, Davison JG, et al. Unit dose calendar packaging and elderly patient compliance. N Z Med J 1991; 104: 495-7.

33. Wong BS, Norman DC. Evaluation of a novel medication aid, the calendar blister-pak, and its effect on drug compliance in a geriatric outpatient clinic. J Am Geriatr Soc 1987; 35: 21-6.

34. Rosen MI, Rigsby MO, Salahi JT, et al. Electronic monitoring and counseling to improve medication adherence. Behav Res Ther 2004; 42: 409-22.

35. Lester RT, Ritvo P, Mills EJ, et al. Effects of a mobile phone short message service on antiretroviral treatment adherence in Kenya (WelTel Kenya1): a randomised trial. Lancet 2010; 376: 1838-45.

36. Krishna S, Boren SA, Balas EA. Healthcare via cell phones: a systematic review. Telemed J E Health 2009; 15: 231-40.

Поступила 24/01-2011 